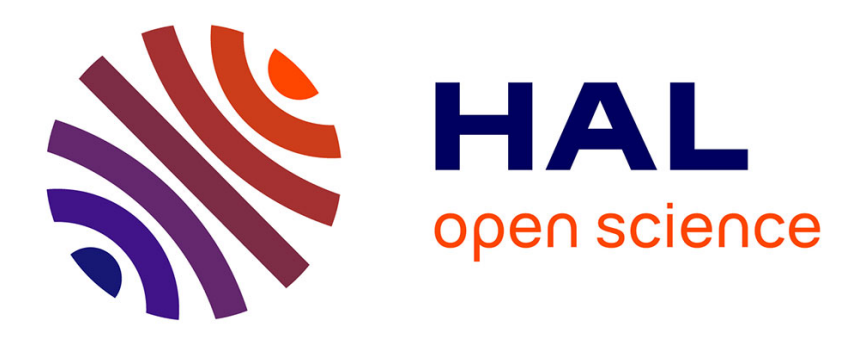

\title{
Dynamical Properties of Wormlike Micelles: A Light Scattering Study
}

E. Buhler, J. Munch, S. Candau

\section{To cite this version:}

E. Buhler, J. Munch, S. Candau. Dynamical Properties of Wormlike Micelles: A Light Scattering Study. Journal de Physique II, 1995, 5 (6), pp.765-787. 10.1051/jp2:1995158 . jpa-00248199

\section{HAL Id: jpa-00248199 https://hal.science/jpa-00248199}

Submitted on 1 Jan 1995

HAL is a multi-disciplinary open access archive for the deposit and dissemination of scientific research documents, whether they are published or not. The documents may come from teaching and research institutions in France or abroad, or from public or private research centers.
L'archive ouverte pluridisciplinaire HAL, est destinée au dépôt et à la diffusion de documents scientifiques de niveau recherche, publiés ou non, émanant des établissements d'enseignement et de recherche français ou étrangers, des laboratoires publics ou privés. 
Classification

Physics Abstracts

$36.20-\mathrm{r}-61.25 \mathrm{Hq}-82.70-\mathrm{y}$

\title{
Dynamical Properties of Wormlike Micelles: A Light Scattering Study
}

\author{
E. Buhler, J.P. Munch and S.J. Candau \\ Laboratorre d'Ultrasons et de Dynamique des Fluides Complexes $\left({ }^{*}\right)$, Université Louis Pasteur, \\ 4 rue Blaise Pascal, 67070 Strasbourg Cedex, France
}

(Received 18 January 1995, accepted 13 March 1995)

\begin{abstract}
Résumé. - Des expériences de diffusion statique et dynamique de la lumière ont été réalisées sur des solutions aqueuses micellaires de bromure d'hexadecyltrimethylammonium (CTAB) en présence de bromure de potassium (KBr) en fonction de la fraction volumique de tensio-actif, de la température et de la teneur en sel. Cette étude confirme l'analogie entre le comportement en régime semi-dilué de systèmes de micelles géantes et des polymères en solution. En particulier, la fonction d'autocorrélation temporelle du champ électrique diffusé est bimodale. Les résultats relatifs au processus lent de relaxation peuvent être comparés aux prédictions de modèles théoriques décrivant le couplage entre les fluctuations de concentration et la viscoélasticité. En particulier le temps caractéristique de la relaxation lente viscoélastique varie avec la température, la teneur en sel et la concentration en tensio-actif de la même manière que le temps terminal de la relaxation de contrainte mesuré par des expériences classiques de rhéologie.
\end{abstract}

\begin{abstract}
Statıc and Dynamic Light Scatterıng experiments were carried out on aqueous micellar solutions of hexadecyltrimethylammonum bromide (CTAB) in the presence of potassium bromide $(\mathrm{KBr})$ as a function of surfactant volume fraction, temperature and salt content. This study confirms strikingly the analogy between the behaviour in semi-dilute regime of elongated micellar systems and polymer solutions. In particular, the time autocorrelation function of the scattered field is found to be bimodal. The results concerning the slow relaxation process can be accounted for by the theoretical models describing the coupling of concentration fluctuations to viscoelasticity. In particular the behaviour of the slow viscoelastic time as a function of temperature, salt content and surfactant concentration parallels that of the terminal time of the stress relaxation measured by a conventional rheological apparatus.
\end{abstract}

\section{Introduction}

In some systems, the micelles present in aqueous solutions of surfactants can undergo uniaxial growth upon an increase of surfactant concentration and/or upon addition of salt [1,2]. When

(*) Unité de Recherche Associée au CNRS No. 851 
this unidimensional growth is highly favored by an appropriate choice of the surfactant/salt couple, the micelles become very long rod-like aggregates which, on a length scale larger than the persistence length, are flexible and resemble polymer molecules [3-9].

The existence of polymer-like phases associated with the formation of long flexible cylinders has been predicted by Safran et al. [10]. In the last years, numerous experimental studies have enforced the analogy between polymers and worm-like micelle solutions $[1,2]$.

The strongest evidence in favor of very flexible micelles was obtained in studies performed in the semi-dilute regime, i.e., at surfactant concentration large enough that the elongated micelles overlap, forming a transient network $[1,2]$. In this regime, the systems exhibit a viscoelastic behaviour, very reminiscent of that of entangled polymer solutions [11-26]. However, there are two major differences between polymer solutions and worm-like micellar systems. The first difference is related to the self-assembling nature of the process of formation of elongated micelles, that leads to equilibrium polymers with a broad distribution of micellar sizes $[1,27,28]$. The average micellar length $\bar{L}$ grows with increasing surfactant volume fraction $\phi$ and salt content. For neutral or highly screened systems, the growth is characterized by a simple power law increase with exponent $1 / 2$. The second difference is due to the fact that the worm-like micelles can break and recombine on a time scale which is dependent on the system and on the physicochemical conditions.

One expects then a solution of semi-dilute worm-like micelles to behave as an entangled network of polymer molecules if observations are made on a time scale shorter than the time $\tau_{\text {break }}$ for scission or recombination. In this case, the properties of the system depend only on the correlation length $\xi$ that can be visualized as the mesh size of the transient network of overlapping cylindrical micelles. These properties can be characterized through the measurement of the intensity of scattered light $I_{\mathrm{s}}$ and of the cooperative diffusion coefficient $D_{\mathrm{c}}$ which are both associated with collective modes of the network, and the high frequency elastic moduli. All these parameters are insensitive to the overall length of the micelles and to the micellar kinetics. Previous experimental studies have shown that $I_{\mathrm{s}}$ and $D_{\mathrm{c}}$ obey power laws of the surfactant volume fraction with exponents close to those predicted for semi-dilute polymer solutions $[4-6,29]$.

At time scales longer than $\tau_{\text {break }}$, the reversible breaking down of elongated micelles has an important influence on the dynamic properties of these systems [2]. Cates has extended to equilibrium polymers the reptation theory which describes the rheological properties of unbreakable chains in the entangled regime [30-32]. Cates's model predicts several rheological regimes depending on the relative rates of diffusive polymer motion and reversible breakdown processes. In particular, a nearly single exponential stress decay function is predicted in the linear viscoelastic response, in the limit where the micelle breaking time is short compared to the reptation time $\tau_{\text {rep }}$ of the micelle of mean length. Deviations from this behaviour appear at a circular frequency $\omega$ of the order of the inverse of $\tau_{\text {break }}$, due to the occurrence of breathing and Rouse modes [33].

A large number of rheological experiments has been performed recently on worm-like solutions and a good agreement was observed between theoretical predictions and experimental results on several systems $[1,2]$. However, significant deviations from this picture were observed in the limit of very high salt concentrations. In particular, it was shown that micellar solutions of hexadecyltrimethylammonium bromide (CTAB) [24] and hexadecylpyridinium chlorate $\left(\mathrm{CPClO}_{3}\right)[25,34,35]$ in the presence of an excess of $\mathrm{KBr}$ and $\mathrm{NaClO}_{3}$, respectively, even though they form extremely long worm-like micelles exhibit an amazingly high fluidity and also a weak dependence of the viscosity on $\phi$, that cannot be accounted for by the coupled reptation-reaction model of Cates [30-33]. It was suggested that this anomalous behaviour was associated with the formation of crosslinks by fusion of micelles, leading to a solution 
of branched micelles. A recent model of reptation of polymers undergoing reversible scission shows that the zero-shear viscosity and its concentration dependence are reduced with respect to those of entangled linear micelles [36]. Contrary to the case of ordinary branched polymers where the distribution of branch points in a molecule is quenched, here the connections can move along the cylindrical part of the micelles, which speeds up the diffusion of the micelle within the tube.

Another approach to the dynamical properties of polymeric systems rests on the analysis by dynamic light scattering of the long time behaviour of the correlation function of the concentration fluctuations. It was shown theoretically that when the coupling of the concentration fluctuations to the viscoelasticity is present, the time correlation function of the concentration fluctuations is bimodal, consisting of a single-exponential decay characterizing the cooperative diffusion and a distribution of relaxation modes associated with the relaxation of the longitudinal stress tensor of the binary solution [37]. The amplitude of the slow component in the spectrum of scattered light becomes more pronounced as the solvent quality is decreased. Experimentally it is found that, although the cooperative diffusion process dominates, slow modes are present in semi-dilute solutions in theta solvent [38]. There has been some controversy about the origin and the concentration dependences of the amplitude and of the average relaxation time associated with these modes. However the experiments of Adam and Delsanti [39] provide strong arguments in favour of the scheme proposed by Brochard and de Gennes, based on a transient gel model [40]. In the case of polymer solutions in good solvent, it seems well accepted now that there is no slow mode in the spectrum of scattered light [38]. However, it must be mentioned that the amplitude of such a mode is very small under good solvent conditions. In solutions of worm-like micelles, bimodal time correlation functions of scattered light have been reported recently by Brown et al. [41].

In this paper, we report light scattering experiments performed on aqueous micellar solutions of $\mathrm{CTAB}$ in the presence of $0.5 \mathrm{M} \mathrm{KBr}$ for concentrations ( $\mathrm{w} / \mathrm{v}$ ) ranging from $0.01 \%$ to $15 \%$ and at temperatures from $30^{\circ} \mathrm{C}$ to $45^{\circ} \mathrm{C}$. Also, experiments were performed on a CTAB solution at $c=0.1 \mathrm{~g} / \mathrm{cm}^{3}$ as a function of the $\mathrm{KBr}$ concentration $(0.5$ to $2.5 \mathrm{M})$. It is shown that the time correlation function of the concentration fluctuations is bimodal. The rate constant of the fast mode is equal to $q^{2} D_{\text {c }}$ where $q$ is the amplitude of the scattering wave vector. The slow mode is $q$-independent. The experimental variations of the relaxation time with surfactant concentration and temperature are compared to those of the terminal time measured by means of rheological experiments. This comparison allows one to conclude on the viscoelastic origine of the slow mode.

\section{Theoretical Background}

2.1. MicellaR Kinetics. - Detailed results concerning the stress decay function were obtained from a computer simulation by Turner and Cates [42] and from a Poisson renewal model by Granek and Cates [33] for various values of the ratio $\zeta=\tau_{\text {break }} / \tau_{\text {rep }}$. The assumptions made for the calculation of the complex shear modulus $G^{*}(\omega)$ are the following:

Firstly, it is assumed that when a chain breaks, the two daughter chains become uncorrelated and evolve separately. This implies that each micellar end recombines with a randomly chosen micellar end and not with the original partner end.

The second assumption is that the only mode of stress relaxation is a reptation process.

The chemical relaxation process is the reversible unimolecular scission, characterized by a temperature-dependent rate constant $k$ per unit time per unit arc length, which is the same for all elongated micelles and is independent of time and of volume fraction. 
Such assumptions are strictly valid in the entangled regime when reaction rates are determined by the local motion of chain subsections and not the diffusion of polymers over distances large compared to their radii of gyration. The micelle breaking time $\tau_{\text {break }}$ is found to be given by:

$$
\tau_{\text {break }}=(k \bar{L})^{-1} \sim k^{-1} \phi^{-1 / 2} \exp \left[-E_{\text {sciss }} / 2 k_{\mathrm{B}} T\right]
$$

where $k_{\mathrm{B}}$ is the Boltzmann constant, $T$ the absolute temperature and $E_{\text {sciss }}$ is the scission energy of the micelle that represents the excess free energy for a pair of hemispherical endcaps relative to a rodlike region containing an equal number of surfactants $[1,10,30-32]$. The above relationships have been derived for nonionic micelles or ionic micelles at large ionic strength.

2.2. Stress Relaxation. - In the semidilute range, i.e., at surfactant concentration large enough for the elongated micelles to overlap, the systems exhibit a viscoelastic behaviour very reminiscent of that of transient polymeric networks $[1,2]$. In polymeric systems, the viscoelastic properties are described by a model based on the reptation theory [43-45]. However, the "living" character of the micelles provides additional pathways for disentanglement. Several regimes of behaviour are predicted depending on the relative values of $\tau_{\text {rep }}$ and $\tau_{\text {break }}$ of a polymeric micelle with a length equal to the average micellar length $\bar{L}$. When $\tau_{\text {break }}$ is long compared to $\tau_{\text {rep }}$, the theory of reptation of polydisperse polymers should apply, leading to a strongly nonexponential stress relaxation function $\mu(t)$ given by:

$$
\mu(t) \sim \exp \left[-a^{\prime}\left(t / \tau_{\text {rep }}\right)^{1 / 4}\right]
$$

where $a^{\prime}$ is a constant.

In the opposite case where $\tau_{\text {break }} \ll \tau_{\text {rep }}$, the micelle breaking plays an important role in the viscous flow process as first stressed by Hoffmann et al. [11-14]. The model of Cates predicts an almost pure exponential form of the stress relaxation function with the terminal time [30].

$$
T_{\mathrm{R}} \sim\left(\tau_{\text {break }} \tau_{\text {rep }}\right)^{1 / 2}
$$

A single exponential stress decay has been indeed reported in many studies on different surfactant systems $[1,2]$.

Later, the renewal model has been used to analyze in detail the crossover to a rapid scission regime in which chain diffusion between scission events is dominated by breathing modes. The variation as a function of the circular frequency $\omega$ of the complex shear modulus $G^{*}(\omega)$ can then be described with two parameters: the ratio $\zeta=\tau_{\text {break }} / \tau_{\text {rep }}$ and the ratio $l_{\mathrm{e}} / \bar{L}$ of the entanglement length $l_{e}$ over the average size $\bar{L}$.

Typical Cole-Cole plots representing parametrized variations of the storage modulus $G^{\prime}(\omega)$ as a function of the loss modulus $G^{\prime \prime}(\omega)$ are given in Figure 1 for $l_{\mathrm{e}} / \bar{L}=0.1$ and for different values of $\zeta(0.1,1$ and 10$)$.

It is seen that the Rouse part of the spectrum leads to a strongly increasing $G^{\prime \prime}$ at high frequency, thus creating a clear dip in the Cole-Cole plot. It was found that provided $\tau_{\text {break }} \gg \tau_{\mathrm{e}}$ the value of $G^{\prime \prime}$ at the dip obeys [33]

$$
G_{\min }^{\prime \prime} / G_{\infty}^{\prime}=A l_{\mathrm{e}} / \bar{L}
$$

Where $\tau_{\mathrm{e}}$ is the Rouse time of an entanglement length $l_{\mathrm{e}}, G_{\infty}^{\prime}$ is the plateau modulus and A is a constant of the order of 1 . For very small $\tau_{\text {break }}$ (e.g., a small multiple of the entanglement time $\tau_{\mathrm{e}}$ ) a large departure from Maxwell behaviour is seen, due to the increasing influence of the Rouse regime at high frequency, but then $G_{\min }^{\prime \prime}$ does not have the same meaning. 


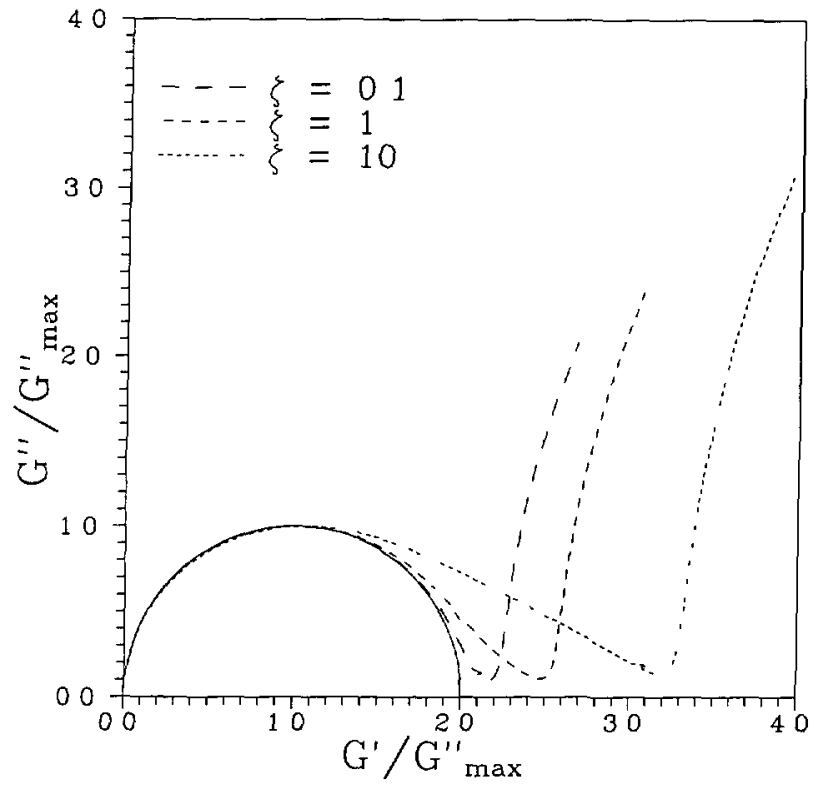

Fig. 1 - Calculated Cole-Cole plots from reference [33] for three values of $\zeta$. The imaginary and real parts of the shear modulus have been normalized by $G_{\max }^{\prime \prime}$, the maximum of $G^{\prime \prime}$.

2.3. Dynamic Light Scattering. - We first recall the main conclusions of the theoretical studies on the normalized dynamical correlation function $g^{(1)}(q, t)$ of polymer concentration fluctuations in semidilute solutions of unbreakable polymers, defined as

$$
g^{(1)}(q, t)=\frac{<\delta c^{*}(q, 0) \delta c(q, t)>}{\left\langle|\delta c(q, 0)|^{2}\right\rangle}
$$

Where $\delta c(q, t)$ and $\delta c(q, 0)$ represent a fluctuation of polymer concentration of wave vector $q$ at time $t$ and zero, respectively, and $q$ is given by:

$$
q=\left(4 \pi n / \lambda_{0}\right) \sin (\theta / 2)
$$

where $n$ is the refractive index of the solution, $\lambda_{0}$ the wavelength in the vacuum and $\theta$ the scattering angle.

Using a transient gel model, de Gennes developed a scaling theory for semidilute solutions in thermodynamically good solvents $[46,47]$. The main prediction of the theory is that the dynamical behaviour of the solution can be described in terms of a single characteristic length, the correlation length $\xi$. As a consequence, the time correlation function of the concentration fluctuations decays according to a purely exponential relaxation characterized by a gel diffusion coefficient

$$
D_{\mathrm{g}}=\frac{M_{\mathrm{g}}+K}{K} D_{\mathrm{c}}
$$

where $M_{\mathrm{g}}$ and $K$ are the gel modulus and the osmotic modulus, respectively, and $D_{\mathrm{c}}$ is the cooperative diffusion coefficient given by:

$$
D_{\mathrm{c}}=\frac{k_{\mathrm{B}} T}{6 \pi \eta_{0} \xi_{\mathrm{H}}}
$$


where $\xi_{\mathrm{H}}$ is the hydrodynamic correlation length that scales like the static correlation length $\xi$ and $\eta_{0}$ is the solvent viscosity.

The correlation length $\xi$ and consequently the cooperative diffusion coefficient $D_{\mathrm{c}}$ follow simple scaling laws to dilution according to

$$
\xi \sim c^{-0.77} \text { and } D_{\mathrm{c}} \sim c^{0.77}
$$

The above behaviour is expected to be observed only in the limit of infinite molecular weight of polymer molecules. In fact, different experimental studies show that $D_{c}$ follow a power law to dilution with exponents varying between 0.6 and 0.8 depending on the system [38].

The above result is obtained under the assumption that the relaxation time $\tau_{\text {rep }}$ for complete disentanglement of one chain from the others is much longer than the time range explored in dynamic light scattering. At low frequency the gel modulus becomes negligible in equation (7) and $D_{\mathrm{g}}$ becomes equal to $D_{\mathrm{c}}$. Later on, the theoretical study was extended to the case of semidilute solutions in theta-solvent [40] and of polymer mixtures [48-50]. It was shown that, whereas in the long-wavelength, low frequency limit, $g^{(1)}(q, t)$ was still a simple exponential decaying as $\exp \left(-D_{\mathrm{c}} q^{2} t\right)$, above a small threshold $q_{\mathrm{g}} \propto N_{\mathrm{e}} N^{-3 / 2}\left(N\right.$ and $N_{e}$, being the numbers of links per chain and per entanglement length, respectively), two relaxational modes were predicted: a fast gel mode with the characteristic time

$$
\tau_{\mathrm{f}}=\frac{1}{D_{\mathrm{g}} q^{2}}
$$

and a slow hydrodynamic mode with the time

$$
\tau_{\mathrm{s}} \simeq \tau_{\mathrm{rep}} \frac{D_{\mathrm{g}}}{D_{\mathrm{c}}}
$$

The above predictions have been experimentally verified by Adam and Delsanti [39]. They observed for semidilute polystyrene solutions in cyclohexane a $q^{2}$-dependent collective diffusion mode and a single $q$-independent relaxation viscoelastic mode. Later on, several other theoretical studies were published on this subject. Wang used a hydrodynamic theory to take into account the coupling of the concentration fluctuations to the viscoelasticity and showed that the model could be used to describe the dynamical behaviour of any viscoelastic liquid, the slow relaxation time being then the stress relaxation time [51]. Doi and Onuki presented phenomenological hydrodynamic equations for entangled polymer blends as generalization of those for polymer solutions [52]. Semenov derived a full treatment where the relaxation of concentration fluctuations takes into account both deformation of the entanglement network and the direct effect of the reptation motion of polymer chains [37]. In reference [40], only the first effect was considered, leading to the prediction given by equation (11).

In Semenov's approach, three stages of relaxation of concentration fluctuations are predicted. The first stage is due to cooperative deformation of entanglement network and is characterized by a time $\tau_{\mathrm{f}}^{-1} \propto q^{2} N^{0}$. The second stage is a Rouse-type relaxation of the macromolecular tension along the tube with a relaxation time $\tau_{\mathrm{R}}$. The last stage associated with the reptation process is characterized by a spectrum of relaxation times between $\tau_{\text {rep }}$ and $T_{\mathbf{R}} ; T_{\mathbf{R}}$ is the terminal time of the stress relaxation function and is proportional to $N^{3}$, and $\tau_{\text {rep }}$ depends on $q$ and $\mathrm{N}$ on a complicated way depending on the relative value of the size of the macromolecule with respect to the inverse of the scattering vector. The variation of the three characteristic relaxation times $\tau_{\mathrm{f}}, \tau_{\mathrm{R}}$ and $\tau_{\text {rep }}$ as a function of $q^{-1}$, as calculated by Semenov is reported in Figure 2. The $q^{-1}$-domain investigated in our experiments extends on both sides of the value 


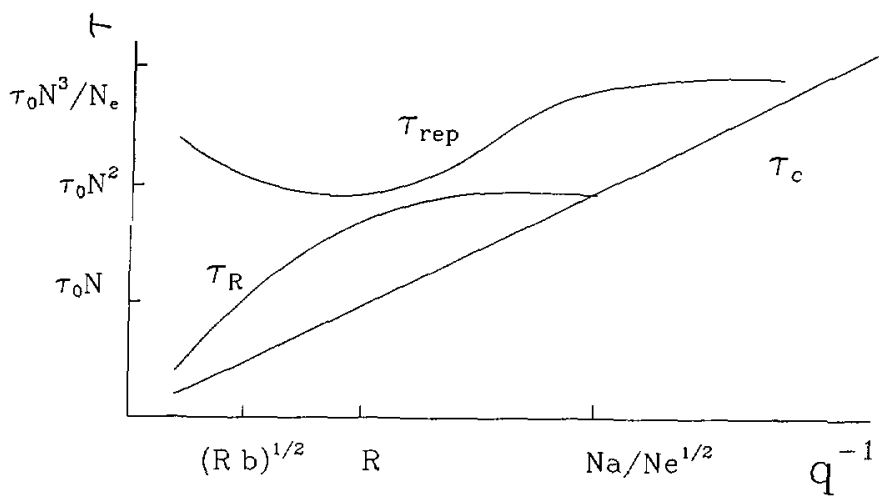

Fig. 2. - Schematic (double logarithmic) plot of cooperative $\left(\tau_{\mathrm{c}}\right)$, Rouse $\left(\tau_{\mathrm{R}}\right)$ and reptational $\left(\tau_{\mathrm{rep}}\right)$ relaxation times versus $q^{-1}, b$ is the tube diameter, and $a$, the length of the statistical unit (from Ref. [37]).

$q^{-1}=R$ ( $R$, end-to-end distance), in a range where, $\tau_{\text {rep }}$ is roughly $q$-independent and vary as:

$$
\tau_{\text {rep }} \sim \tau_{0} N^{2} q^{0}
$$

where $\tau_{0}$ is the characteristic microscopic time. Semenov has also calculated the amplitudes of the different relaxational contributions. As a rule, the amplitude of the first cooperative stage $A_{\mathrm{f}}$ is the largest, and the amplitude $A_{\mathrm{R}}$ of the second "Rouse" stage is the smallest. Considering the reduced amplitudes of the three relaxation stages and the corresponding normalized correlation function $g_{\mathrm{f}}(t), g_{\mathrm{R}}(t)$ and $g_{\mathrm{rep}}(t)$ with $g_{\mathrm{f}}(0)=g_{\mathrm{R}}(0)=g_{\mathrm{rep}}(0)=1$ and $A_{\mathrm{c}}+A_{\mathrm{R}}+A_{\mathrm{rep}}=1$, we find the following relationships:

$$
\begin{array}{rlr}
A_{\mathrm{c}} & =\frac{K}{M_{\mathrm{g}}+K} & \\
A_{\mathrm{R}} & \simeq \frac{1}{6} \frac{M_{\mathrm{g}}}{K} \quad \text { for } q R \gg 1 \\
A_{\text {rep }} & \simeq \frac{5}{6} \frac{M_{\mathrm{g}}}{K}
\end{array}
$$

and

$$
\begin{aligned}
A_{\mathrm{c}} & =\frac{K}{M_{\mathrm{g}}+K} \\
A_{\mathrm{R}} & \simeq \frac{7}{15} \frac{M_{\mathrm{g}}}{K} \quad \text { for } q R \ll 1 \\
A_{\text {rep }} & \simeq \frac{8}{15} \frac{M_{\mathrm{g}}}{K}
\end{aligned}
$$

In good solvent conditions $M_{\mathrm{g}} / K \ll 1$, in which case the cooperative diffusion process dominates. Typically, $M_{\mathrm{g}} / K$ is of the order of $5 \times 10^{-2}$ and this explains why the slow mode is difficult to detect in semi-dilute solutions of "classical polymers". On the other hand, in theta solvent where $M_{\mathrm{g}} / K \sim 1$, the amplitude of the slow mode can become comparable to that of the fast one. As for the Rouse mode, its amplitude in the regime $q R \gg 1$ is negligible, whereas in the low $q$ range, it becomes comparable to that of the reptational time. However it can be seen in Figure 2 that the relaxation times associated with Rouse-like and reptational 
processes are also rather close, so that it seems very difficult to separate experimentally these two contributions.

Turning now to the worm-like micelles, one expects to observe a behaviour qualitatively similar with a fast cooperative mode and a $q$-independent slow viscoelastic relaxation. The latter should be controlled by the stress relaxation process described in the preceding paragraph. In particular we should observe a single exponential behaviour in the long time scale, followed by deviations from exponentiality at times of the order of $\tau_{\text {break }}$ and eventually a mode corresponding to the Rouse relaxation. Inspection of Figure 1 shows that we can characterize a typical frequency $\omega_{c}$ and therefore a characteristic time $t_{c}=1 / 2 \pi \omega_{c}$ beyond which the stress relaxation can be approximated by a single exponential. This will be used in our analysis of the experimental data.

\section{Materials and Methods}

The CTAB preparation, purification and characterization have been reported [53].

For light scattering measurements, the CTAB solutions were filtered through $0.22 \mu \mathrm{m} \mathrm{Milli-}$ pore filter into the cylindrical scattering cell. The scattering vector $q$ ranges from $3 \times 10^{-4}$ to $3 \times 10^{-3} \AA^{-1}$. In Dynamic Light Scattering (DLS) experiments, we measure the normalized time autocorrelation function $g^{(2)}(q, t)$ of the scattered intensity:

$$
g^{(2)}(q, t)=\frac{\left\langle I^{*}(q, 0) I(q, t)\right\rangle}{\left\langle I(q, 0)^{2}\right\rangle}
$$

The latter can be expressed in terms of the field autocorrelation function or equivalently to the autocorrelation function of concentration fluctuations $g^{(1)}(q, t)$ through:

$$
g^{(2)}(q, t)=1+\beta\left|g^{(1)}(q, t)\right|^{2}
$$

$\beta$ is a coherence factor which in our experiments is equal to $0.90-0.98$.

Static Light Scattering (SLS) and Dynamic Light Scattering (DLS) experiments were performed by means of a spectrometer equipped with an argon ion laser $\left(\lambda_{0}=488 \mathrm{~nm}\right)$, an ALV 5000 correlator and a computer controlled and stepping motor driven variable angle detection system. In SLS experiments, we measure the excess of scattered intensity $I(q)$ with respect to the solvent. No correction into absolute scattering intensities $\Delta R(q)$ (i.e, excess Rayleigh ratio) were made, as we were only interested in relative variations of the scattering intensity.

The rheological experiments were performed on a Carrimed CSL apparatus (controlled stress). We used a plane-plane geometry, the diameter of the planes being $40 \mathrm{~mm}$. A small amplitude oscillatory shear flow is applied to the investigated sample. From the phase lag of the corresponding shear stress and the ratio of the amplitudes of the imposed oscillation and of the response of the solution are calculated the storage modulus $G^{\prime}(\omega)$ and the loss modulus $G^{\prime \prime}(\omega)$. By plotting $G^{\prime}$ as a function of $G^{\prime \prime}$ we obtain the Cole-Cole plot.

\section{Experimental Results}

\subsection{Data Analysis}

4.1.1. Stress relaxation. - Figure 3 shows typical experimental results relative to the frequency dependence of the complex shear modulus for a CTAB solution at $c=10^{-1} \mathrm{gcm}^{-3}$ in presence of $0.5 \mathrm{M} \mathrm{KBr}$ at $T=30^{\circ} \mathrm{C}$. In the low-frequency range that corresponds to the left side of the Cole-Cole plot, the data fit a half-circle centered on the horizontal axis, characteristic 


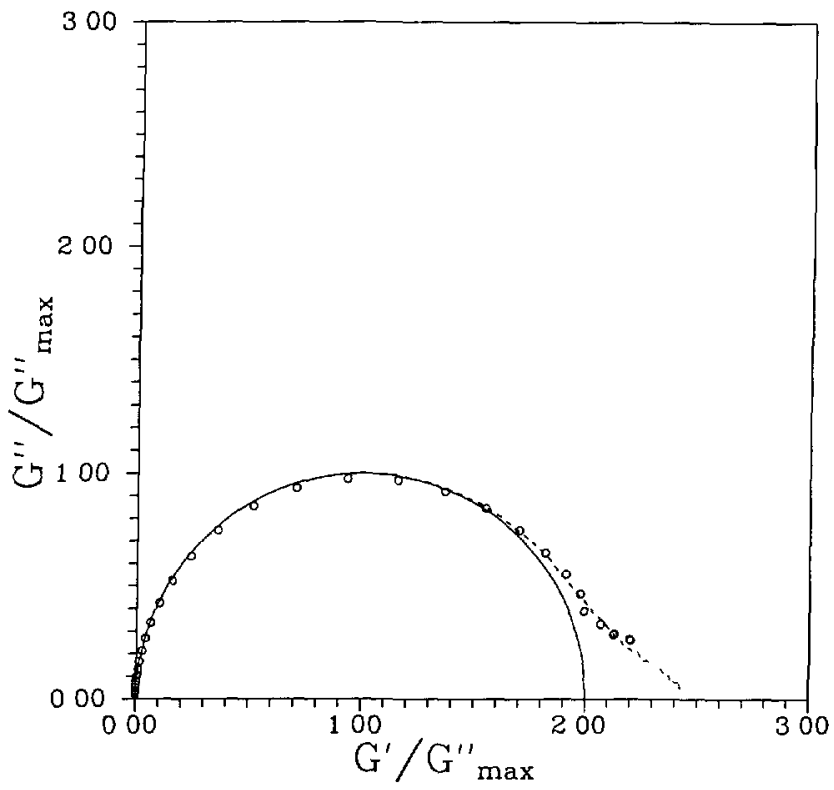

Fig. 3. - Experimental Cole-Cole normalized plot (filled circles). The dotted line represents the calculated Cole-Cole plot disregarding non-reptative effects fitting best the experimental data $(\zeta=0.06)$. Also represented is the osculating semicircle at the origin, $c=0.1 \mathrm{gcm}^{-3},[\mathrm{KBr}]=0.5 \mathrm{M}$ and $T=30^{\circ} \mathrm{C}$.

of a Maxwellian behaviour. At higher frequencies there are deviations from this behaviour that are closely linked to the ratio $\zeta=\tau_{\text {break }} / \tau_{\text {rep. }}$. To determine the ratio $\zeta$ we can use the procedure described in reference [23] based on the superimposition to the experimental data of the theoretical Cole-Cole plots that disregards non-reptative high-frequency effects, calculated for different values of $\zeta$. In Figure 3 we have plotted the osculating circle (full line) at the origin and the theoretical Cole-Cole plot that fits the data best. From such a fit we determine $\zeta=\tau_{\text {break }} / \tau_{\text {rep }}=0.06$.

The low-frequency data or low-shear measurements yield the zero-shear viscosity $\eta$. An estimate of the plateau modulus is provided by $G_{\text {osc }}$ given by the maximum of the osculating circle at the origin. In doing so, we slightly underestimate $G_{\infty}^{\prime}$ that should be measured by extrapolation of the Cole-Cole data to the horizontal axis, but often this extrapolation is delicate, due to the insufficient frequency range explored experimentally.

The stress relaxation time $T_{\mathrm{R}}$ is obtained by combining the values of $\eta$ and $G_{\infty}^{\prime}$ according to:

$$
\eta \sim G_{\infty}^{\prime} T_{\mathrm{R}}
$$

Then by combining the values of $T_{\mathrm{R}}$ and $\zeta$ we can determine both $\tau_{\text {rep }}$ and $\tau_{\text {break }}$. For the case of the $0.5 \mathrm{M} \mathrm{KBr} \mathrm{CTAB}$ solution at $c=0.1 \mathrm{~g} / \mathrm{cm}^{3}$ and $T=30^{\circ} \mathrm{C}$, the results of Figure 3 lead to $\tau_{\text {break }}=0.106 \mathrm{~s}$.

In Figure 3 we do not see the clear dip in the Cole-Cole plots shown in the theoretical plots of Figure 1 and associated with the Rouse part of the spectrum. However, the break observed at high frequency is likely to correspond to the occurrence of Rouse modes. Taking the corresponding value of $G^{\prime \prime}$ as $G_{\min }^{\prime \prime}$ and measuring $G_{\infty}^{\prime}$ by extrapolation of the Cole-Cole data to the horizontal axis, we have $G_{\min }^{\prime \prime} / G_{\infty}^{\prime} \sim 0.1$ that according to equation (4) is also equal to $A l_{\mathrm{e}} / \bar{L}$. 
The entanglement length $l_{\mathrm{e}}$ can be estimated from the relation

$$
G_{\infty}^{\prime}=k_{\mathrm{B}} T / \xi^{3}=k_{\mathrm{B}} T / l_{\mathrm{e}}^{9 / 5}
$$

where it has been assumed that we are under good solvent conditions. From the above equation $l_{\mathrm{e}}$ is estimed to be $372 \AA$ for the sample at $c=0.1 \mathrm{gcm}^{-3}$. By using equation (4) and assuming $A=1$ we obtain $\bar{L}=3720 \AA$. This value must be considered only as an order of magnitude because of both the approximations used in equations (4) and (18) and the uncertainty in the determination of $l_{\mathrm{e}} / \bar{L}$.

Two other parameters that will be relevant for the discussion of the DLS results are the frequency $f_{\max }$ at which $G^{\prime \prime}$ goes through a maximum to which corresponds a characteristic time $\tau_{\max }=1 / 2 \pi f_{\max }$, and also the characteristic time $t_{\mathrm{c}}$ at which the data depart from the osculating circle. The data of Figure 3 lead to $t_{\mathrm{c}} \sim 0.035 \mathrm{~s}$.

4.1.2. Scattered Intensity. - Plots of $c / I(q)$ versus $q^{2}$ were extrapolated to $q=0$ to give intercepts $c / I(0)$. In dilute solution, the average radius of gyration $R_{\mathrm{G}}$ was determined from the intercept and the slope of these plots using a Lorentzian scattering law of the form:

$$
\frac{c}{I(q)}=\frac{c}{I(0)}\left[1+\frac{q^{2} R_{\mathrm{G}}}{3}\right]
$$

In the semidilute regime, a similar expression allows one to determine the static correlation length $\xi$ according to:

$$
\frac{c}{I(q)}=\frac{c}{I(0)}\left[1+q^{2} \xi^{2}\right]
$$

4.1.3. Normalized Time Autocorrelation Function of Scattered Field. - Figure 4 shows $\log -\log$ plots of $g^{(1)}(q, t)$ for $0.5 \mathrm{M} \mathrm{KBr}$ solutions of CTAB at different surfactant concentrations and at $T=33.2^{\circ} \mathrm{C}$. In the low concentration range $g^{(1)}(q, t)$ is a single exponential, whereas at high concentration, the autocorrelation function of scattered field can be described by a sum of two relaxations widely separated in time. As shown later on, the systems for which a single relaxation process is observed turn out to be in the dilute regime, whereas the other systems are semi-dilute solutions. The scattering angle dependence of $g^{(\mathbf{1})}(q, t)$ is illustrated in Figure 5a. Figure $5 \mathrm{~b}$ gives the same correlation function in a representation where the time axis has been multiplied by $\sin ^{2}(\theta / 2)$. The inspection of Figure 5 shows clearly that the fast mode is a diffusive one with characteristic time inversely proportioned to $q^{2}$, whereas the slow mode is of a viscoelastic nature, the characteristic time being independent of $q$. To analyse the data we have used the following procedure. For dilute solutions, characterized by a single relaxation mechanism, we have adopted the classical cumulant analysis [54]. This analysis provides the variance of the correlation function and the first reduced cumulant $\left(\tau q^{2}\right)^{-1}$ where $\tau$ is the average relaxation time of $g^{(1)}(q, t)$. The extrapolation of $\left(\tau q^{2}\right)^{-1}$ to $q=0$ yields the values of the mutual diffusion constant $D$. The latter is related to the average hydrodynamic radius $R_{\mathrm{H}}$ of the micelles through

$$
D=\frac{k_{\mathrm{B}} T}{6 \pi \eta_{0} R_{\mathrm{H}}}
$$

In the semidilute regime, characterized by two distinct relaxation processes, we proceed as follows: first we plot in semi-log coordinates the function $g^{(1)}(q, t)$ obtained by means of equation (16). An example is given in Figure 6 relative to a $0.5 \mathrm{M} \mathrm{KBr}$ solution of CTAB at $c=0.1$ $\mathrm{gcm}^{-3}$. One clearly sees in the long time scale an asymptotic behaviour that can be described to the first approximation by a straight line. This linear behaviour is obtained (for the exemple presented in Fig. 6) at times $t>\sim 10 \mathrm{~ms}$. This corresponds roughly to the time range $t>t_{\mathrm{c}}$ 


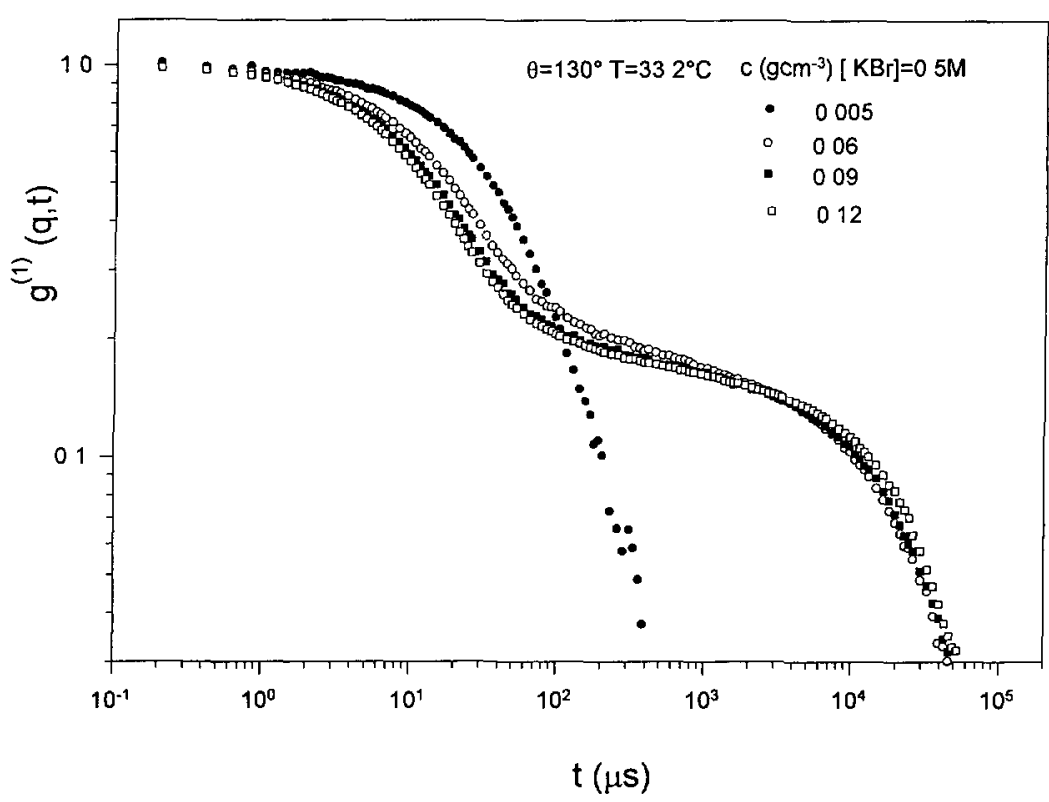

Fig 4. - Log-log representation of $g^{(1)}(q, t)$ for different surfactant concentrations.

for which we observe a semi-circular shape of the Cole-Cole plot in rheological experiments (see preceding paragraph). From the slope and the intercept of the straight lines we can determine the slow relaxation time $\tau_{\mathrm{s}}$ and the corresponding relative amplitude $A_{\mathrm{s}}$. As for the first cumulant associated with the fast mode $\tau_{\mathrm{f}}$, it can be obtained from the initial slope of $g^{(1)}(q, t)$.

Another method to determine $\tau_{\mathrm{s}}$ and $\tau_{\mathrm{f}}$ is the Contin method developed by Provencher [55] and based on the Laplace transform of $g^{(1)}(q, t)$. In the present case, this method can be used quite safely as the two relaxation processes are widely separated in time. If the spectral profile of the scattered light can be described by a multi-Lorentzian curve, then $g^{(1)}(q, t)$ can be written as

$$
g^{(1)}(q, t)=\int_{0}^{\infty} G(\Gamma) \mathrm{e}^{-\Gamma t} \mathrm{~d} \Gamma
$$

where $G(\Gamma)$ is the normalized decay constant distribution. Figure 7 shows a typical example of results obtained by applying the Contin method to our data. We clearly distinguish the slow and the fast modes and in most cases, like in the example of Figure 7 an intermediate mode of small amplitude. The meaning of such a mode will be discussed later. From the fast component of $G(\Gamma)$ we extract the first cumulant. From the slow component we can determine different averages of the slow relaxation times. As long as these relaxation times are not too long we can measure up to 4 averages corresponding to the moments of the distribution of order 0 to 3 and labelled $\tau_{\mathrm{s}}{ }^{(0)}$ to $\tau_{\mathrm{s}}{ }^{(3)}$. It is then found with a quite good accuracy that $\tau_{\mathrm{s}}{ }^{(3)}$ coincides with the relaxation time obtained from the asymptotic behaviour of $g^{(1)}(q, t)$ at long times shown in Figure 6. Thus $\tau_{\mathrm{s}}{ }^{(3)}$ represents the longest time of the distribution. However, as the relaxation times become very long, that is at high surfactant concentration and/or high salt content, the determination of the high moments of the distribution becomes inaccurate with large fluctuations in the values obtained from one experiment to another. In these cases we restrict ourselves to the measurement of $\tau_{s}{ }^{(0)}$. 


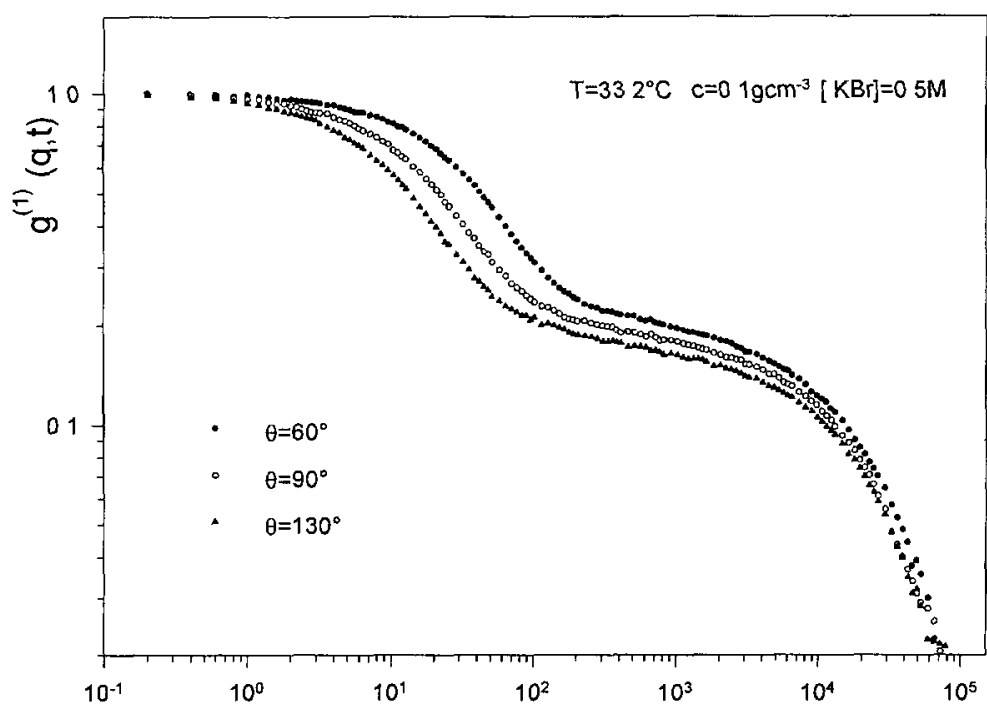

a)

$t(\mu s)$

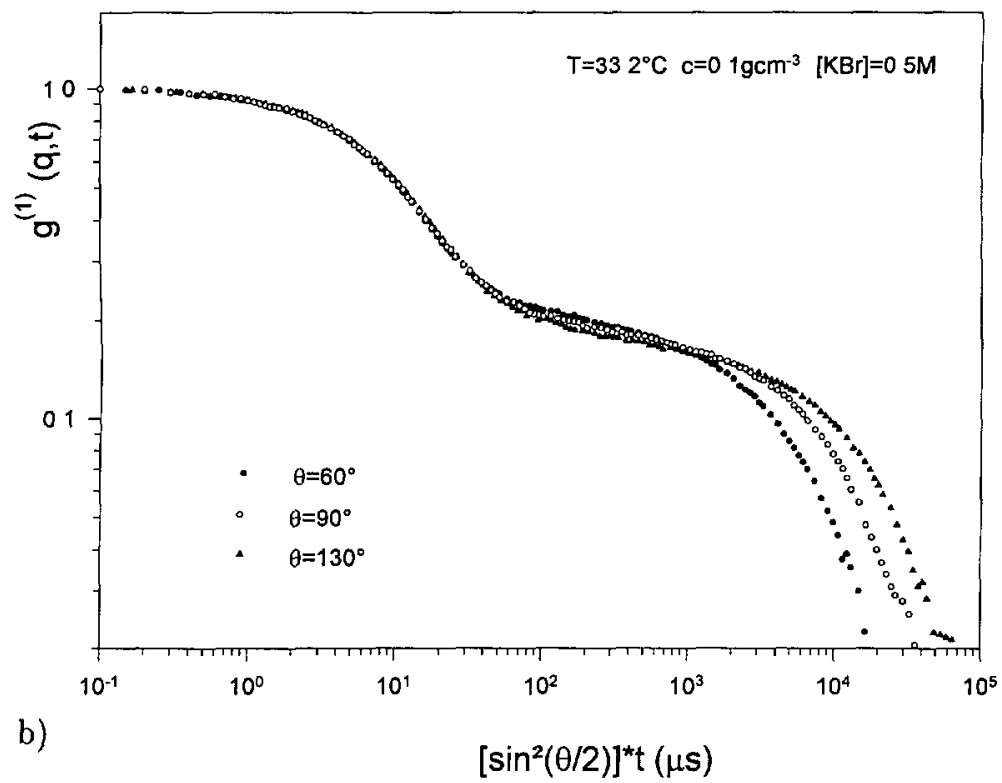

Fig. 5. - a) Time autocorrelation function of the scattered field for different scattering angles. For sake of clarity we have reported only three functions obtained for $\theta \geq 60^{\circ}$; b) Same data as in (a) plotted as a function of $\left[\sin ^{2}(\theta / 2)\right] \times t$.

4.2. Correlation Lengths and Osmotic Compressibility. - Figures 8 show the surfactant concentration dependence of $\xi$ and $\xi_{\mathrm{H}}$ as measured according to the procedure described in the section "Data analysis" for $0.5 \mathrm{M} \mathrm{KBr}$ solutions of CTAB at $T=33.2{ }^{\circ} \mathrm{C}$. One observes the behaviour already reported for other micellar systems [38] that is, first an increase of the correlation lengths, that go through a maximum to decay at high surfactant concentration. 


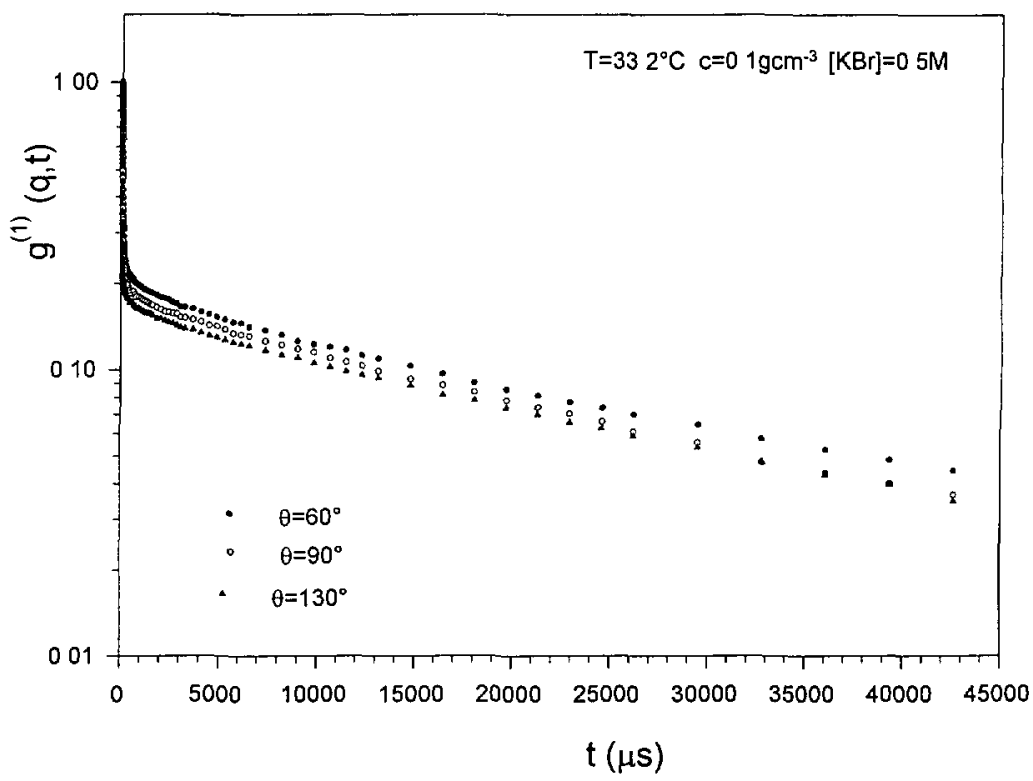

Fig. 6. - Semi-log representation of the time autocorrelation function of the scattered field at different scattering angles.

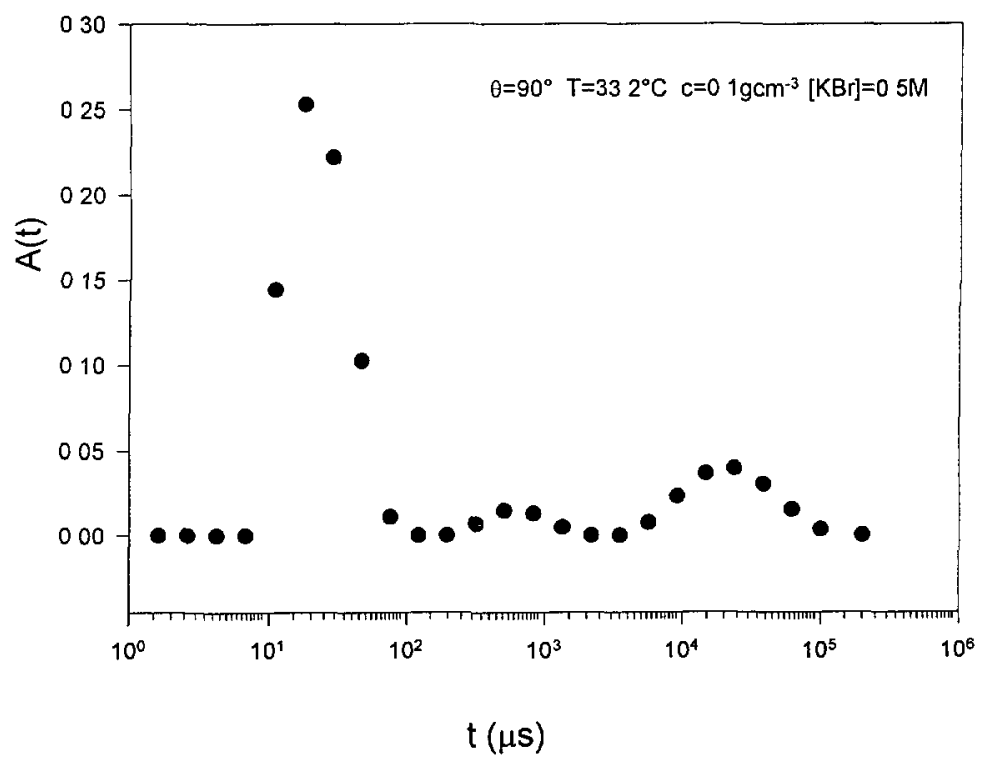

Fig. 7. - Distribution function of decay times $A(t)$ obtained by the Contin method.

The maximum of $\xi$ and $\xi_{\mathrm{H}}$ corresponds to the crossover concentration $c^{*}$ between dilute and semi-dilute solutions. Below $c^{*}$ the chains are separated from each other and the correlation lengths $\xi$ and $\xi_{\mathrm{H}}$ can be identified to the first approximation to $R_{\mathrm{G}} / \sqrt{3}$, the radius of gyration (cf. Eqs. $(19,20)$ ) and $R_{\mathrm{H}}$, the hydrodynamic radius, respectively. Both $R_{\mathrm{G}}$ and $R_{\mathrm{H}}$ increase 
(a)

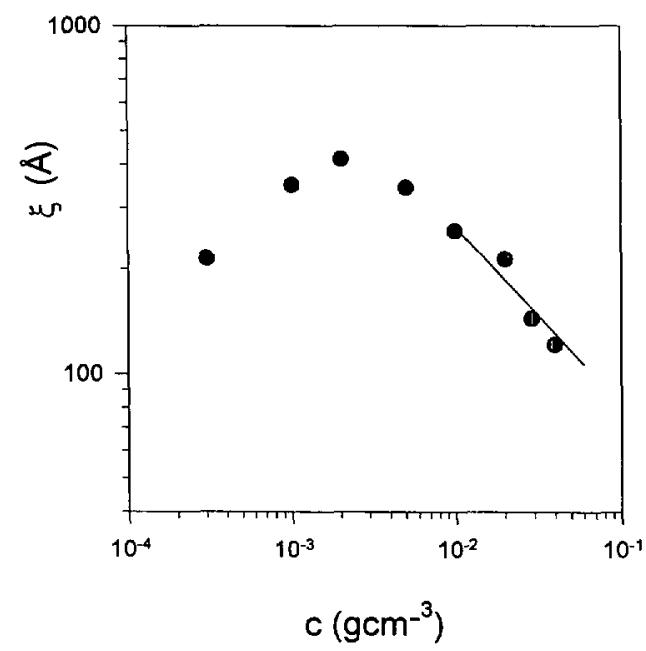

(b)

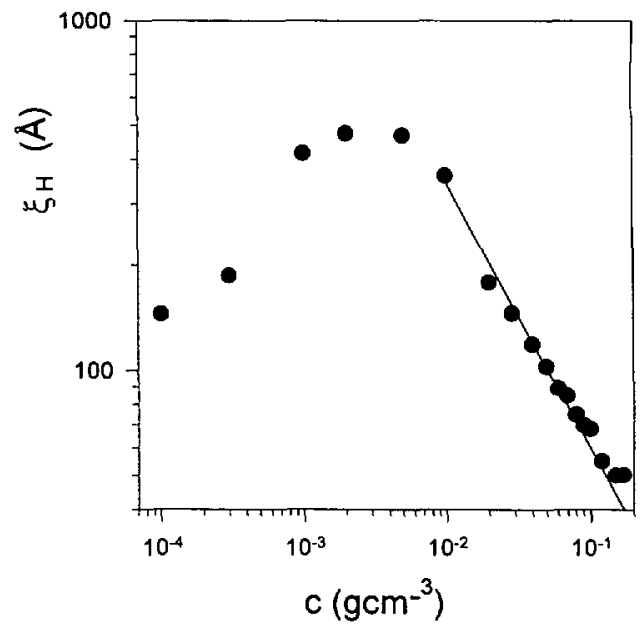

Fig. 8. - Effect of surfactant concentration on a) the static and b) the dynamic correlation lengths. $[\mathrm{KBr}]=05 \mathrm{M}, T=33.2{ }^{\circ} \mathrm{C}$ The straight lines are the best linear fits to the data in the semi-dilute regime.

upon increasing surfactant concentration because $\bar{L} \sim c^{1 / 2}$. Above $c^{*}$ the correlation lengths are correlated to the "blob" size, i.e., the mesh size of the transient network that decreases upon increasing concentration. The behaviour at $c \gg c^{*}$ of $\xi$ and $\xi_{\mathrm{H}}$ is described by power laws $\xi \sim c^{-0.51}$ and $\xi_{\mathrm{H}} \sim c^{-0}{ }^{76}$, respectively. The latter is in good agreement with the theoretical prediction of equation (9) derived for good solvent conditions. As for the static correlation length, the domain of concentration investigated is not large enough to measure the exponent of the power law with a good accuracy. It can also be pointed out that the variance is equal to 0.1 in the dilute regime and decreases significantly in the semidilute regime to the value of 0.01 . This behaviour already reported in previous studies [4-6] can be explained by the large polydispersity in size of the micelles that affects the scattered signal only in the dilute range, since in the semidilute regime one probes collective motions that depend only on $\xi$.

Further information on the concentration dependence of the correlation length is given by the zero- $q$ limit of the scattered intensity. The latter is related to the osmotic modulus through

$$
\Delta R(0)=k^{\prime} K=k^{\prime} c\left(\frac{\partial \Pi}{\partial c}\right)^{-1} k_{\mathrm{B}} T
$$

where $k^{\prime}$ is a constant for a given system $k^{\prime}=4 \pi^{2} n^{2}\left(\frac{\partial n}{\partial c}\right)^{2} \lambda_{0}^{-4}$ and $\Pi$ is the osmotic pressure.

In the dilute regime, one can use a virial expression for the osmotic pressure and equation (23) becomes

$$
\Delta R(0) \simeq k^{\prime} c M_{\mathrm{W}}\left(1-2 A_{2} M_{\mathrm{W}} c+\ldots\right)
$$

where $M_{\mathrm{W}}$ is the weight average molecular weight.

As $M_{\mathrm{W}}$ increases with concentration for surfactant systems, then $\Delta R(0)$ increases also.

In the semi-dilute regime, the scattered intensity becomes independent of the molecular weight of the worm-like micelles and its concentration dependence can be described by a power 


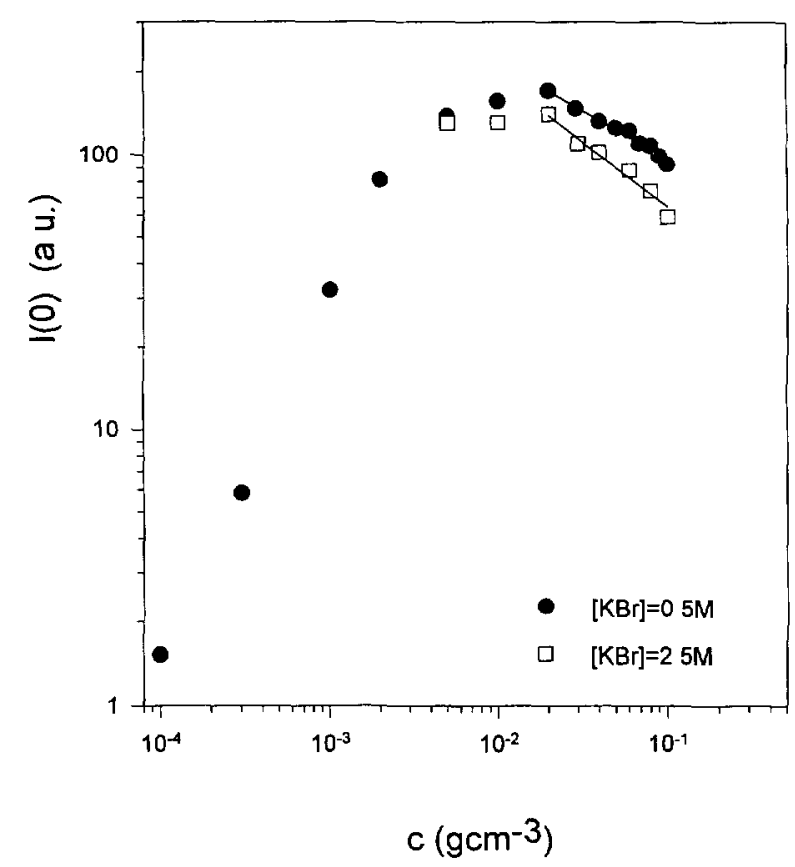

Fig. 9. - Effect of surfactant concentration on the scattered intensity extrapoled at zero- $q$, (•) $[\mathrm{KBr}]=0.5 \mathrm{M} ;(\square)[\mathrm{KBr}]=2.5 \mathrm{M} ; T=332{ }^{\circ} \mathrm{C}$. The straight lines represent the best linear fits to the data in the semi-dilute regime.

law of the form $[38,47,56]$.

$$
\frac{\Delta R(0)}{k^{\prime} c} \sim \phi^{-0.31}
$$

still assuming good solvent conditions.

According to equations (24) and (25), the scattered intensity should go through a maximum, in the vicinity of $c^{*}$. This is what we observe in Figure 9 . In the semidilute regime, the data relative to the $0.5 \mathrm{M} \mathrm{KBr}$ solutions fit a power law with an exponent -0.35 , which within the experimental accuracy is in agreement with the theoretical prediction (Eq. (25)). For the solutions with $2.5 \mathrm{M} \mathrm{KBr}$, the best fit is obtained for a scaling exponent equal to -0.48 . We will discuss later on the behaviour of systems at high salt content. The temperature dependences of the hydrodynamic correlation length for a $0.5 \mathrm{M} \mathrm{KBr}$ solution of CTAB at $c=0.1 \mathrm{gcm}^{-3}$ is reported in Figure 10. One observes a slight decrease of this parameter with temperature. This effect is however within the limits of the experimental accuracy.

The effect of the salt content on the hydrodynamic correlation length and on the zero $q$ scattered intensity is shown in Figures 11 . One observes a net decrease of the scattered intensity upon increasing the salt content whereas the hydrodynamic correlation length exhibits a small minimum at $[\mathrm{KBr}]=2 \mathrm{M}$.

4.3. Linear Viscoelasticity. - In Figure 12a are reported the variations with the temperature of the different moments of the distribution of relaxation times $\tau_{s}{ }^{(1)}$ measured from the Contin analysis for a CTAB solution at $c=0.1 \mathrm{gcm}^{-3}$ in the presence of $0.5 \mathrm{M} \mathrm{KBr}$. In the same figure are reported the relaxation times $\tau_{\max }$ obtained from the maximum of the Cole-Cole plots in rheological experiments. It can be observed that $\tau_{\max }$ is rather close to 


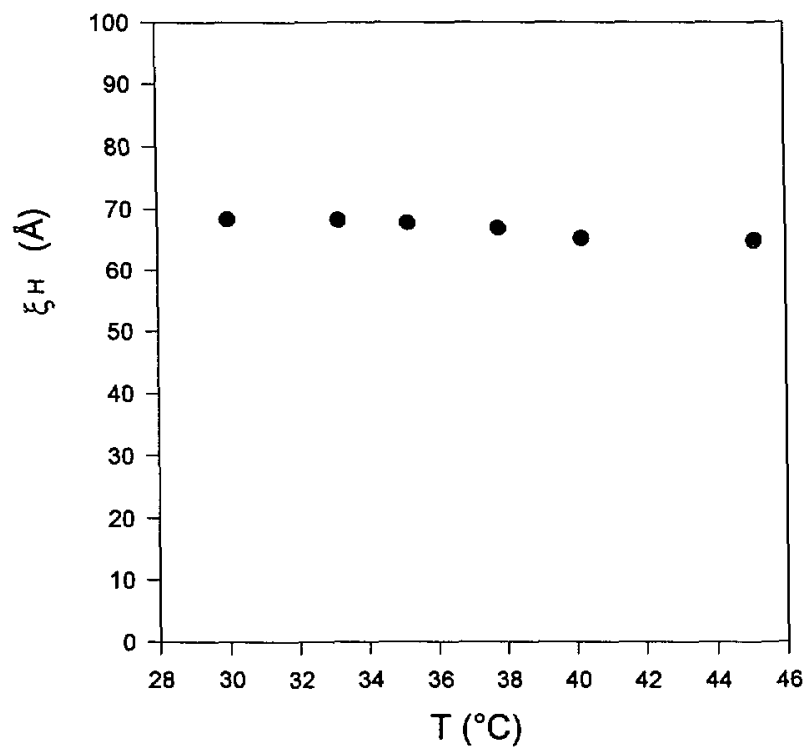

Fig. 10. - Effect of the temperature on the hydrodynamic correlation length. $c=0.1 \mathrm{gcm}^{-3}$, $[\mathrm{KBr}]=0.5 \mathrm{M}$.

(a)

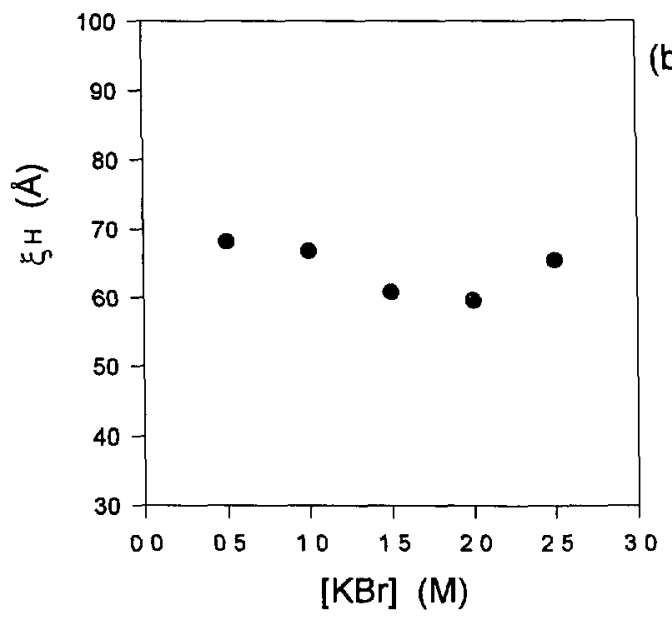

(b)

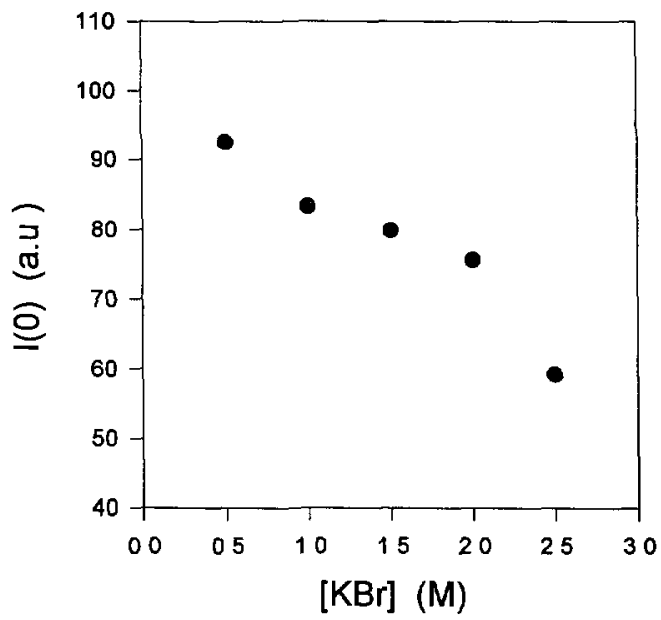

Fig. 11. - Variation with the $\mathrm{KBr}$ concentration of a) the hydrodynamic correlation length and of b) the intensity scattered at zero- $q, c=0.1 \mathrm{gcm}^{-3}, T=33.2^{\circ} \mathrm{C}$.

$\tau_{s}{ }^{(3)}$, that is, the longest time of the distribution. In fact, the different moments $\tau_{s}{ }^{(1)}$ exhibit a similar behaviour as a function of temperature as shown in Figure 12a where have we plotted the variations of $\tau_{s}^{(1)}$ normalized at $T=33.2^{\circ} \mathrm{C}$ Figure $12 \mathrm{~b}$. Figure 13 illustrates the effect of the salt content on both $\tau_{s}{ }^{(0)}$ and $\tau_{\max }$. One observes in both cases a maximum at $2 \mathrm{M} \mathrm{KBr}$. The values of $\tau_{\mathbf{s}}{ }^{(3)}$ are too large to be measured at high $\mathrm{KBr}$ concentration as explained in the section "Data analysis".

In Figure 14 is reported the variation with CTAB concentration of the measurable moments 

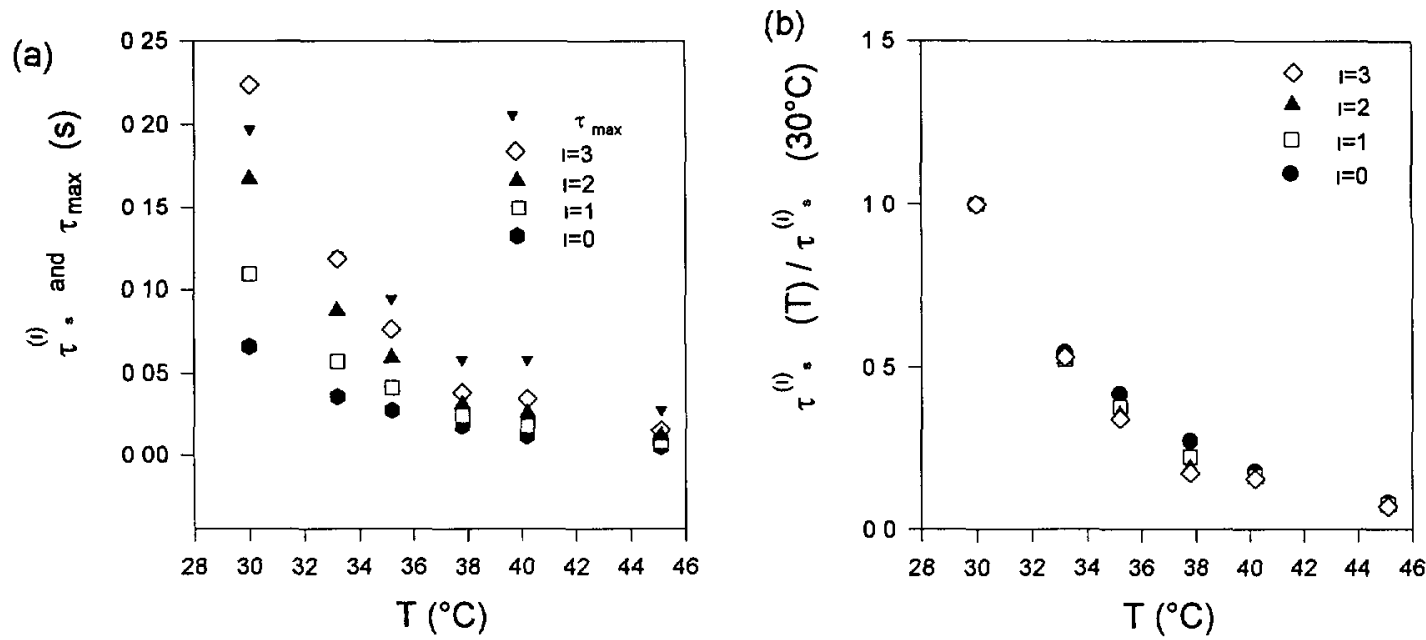

Fig. $12-$ a) Temperature dependence of the terminal time of the stress relaxation and of the moments $\tau_{s}^{(1)}$ of the distribution of relaxation times. In b) are plotted the normalized moments, $c=0.1 \mathrm{gcm}^{-3},[\mathrm{KBr}]=0.5 \mathrm{M}$.

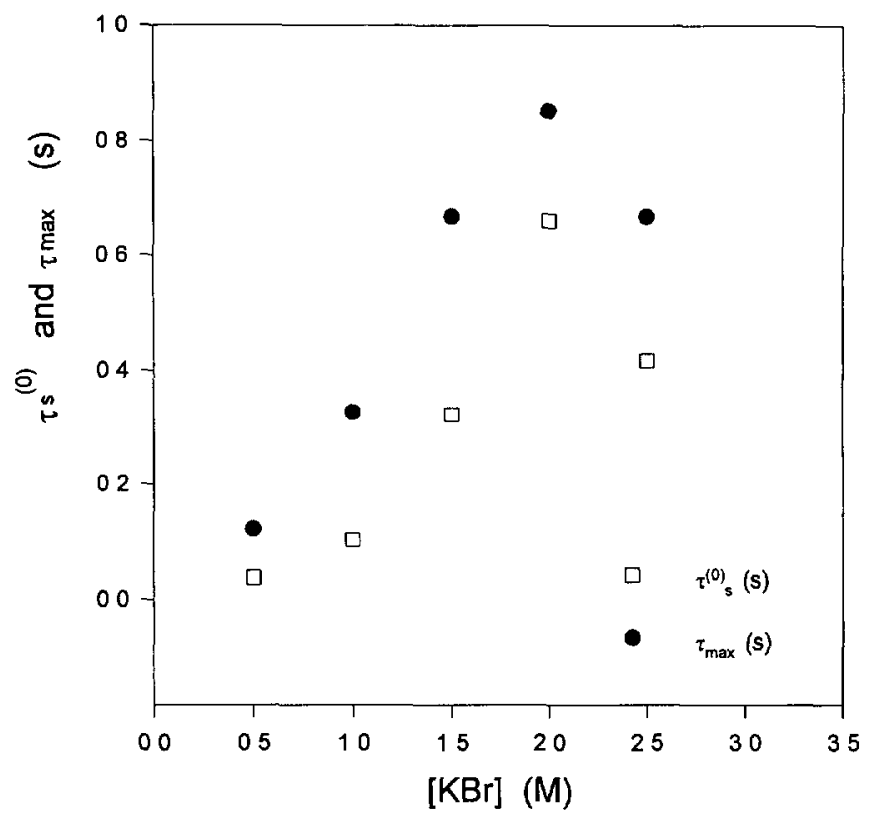

Fig. 13. - Variation of $\tau_{\mathrm{s}}{ }^{(0)}$ and of $\tau_{\max }$ with $[\mathrm{KBr}], c=01 \mathrm{gcm}^{-3}, T=33.2^{\circ} \mathrm{C}$.

$\tau_{\mathrm{s}}{ }^{(1)}$ of the distribution of relaxation times in $0.5 \mathrm{M} \mathrm{KBr}$ solutions. The $\mathrm{c}$ dependence (from 0.09 to $0.15 \mathrm{gcm}^{-3}$ ) of $\tau_{\mathrm{s}}{ }^{(0)}$ can be described by a power law with an exponent 1.03. Scaling laws were reported for the terminal time of the stress relaxation in the same systems at different $\mathrm{KBr}$ contents $[1,24]$. The exponent appears to be dependent on the salt content and the result obtained here is consistent with the previously obtained values. 


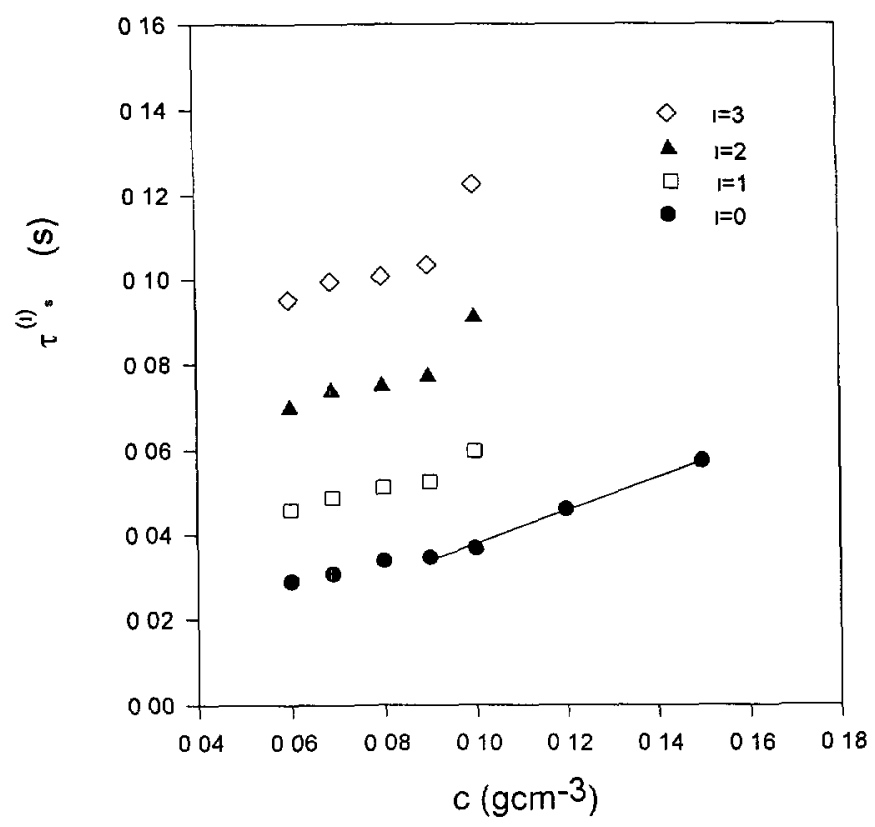

Fig. 14. - Effect of surfactant concentration on $\tau_{s}^{(1)},[\mathrm{KBr}]=0.5 \mathrm{M}, T=33.2{ }^{\circ} \mathrm{C}$.

\section{Discussion}

5.1. Correlation LengthS and Osmotic Compressibility. - The ensemble of the experimental results reported above provides a confirmation of the analogy between worm-like micellar systems and polymer solutions. In this respect, the behaviour of the correlation lengths and of the osmotic compressibility as a function of the surfactant concentration, that was already reported for other systems [1,4-7], is quite characteristic: the maxima of the correlation lengths and of the zero- $q$ scattered intensity observed in Figures 8 and 9 are the signatures of the cross-over between the dilute regime where one probes the micellar size that increases with surfactant concentration and the semi-dilute regime where the scattering is controlled by the correlation length that is a decreasing function of the surfactant concentration. In fact, the comparison of Figures 8 and 9 shows that the maximum of $I_{\mathrm{s}}$ is shifted towards higher concentrations with respect to the maximum of $\xi$ and $\xi_{\mathbf{H}}$. Such a behaviour, already reported $[4-6,57]$, can be explained if we consider the full expression of the zero- $q$ structure factor $S(0)$ for solutions of flexible chains derived by Ohta and Oono on the basis of conformation space renormalization group theory [58]:

$$
S(0)^{-1}=1+\frac{1}{8}\left[9 x-2+\frac{2 \ln (1+x)}{x}\right] \exp \left[\frac{1}{4}\left(\frac{1}{x}+\left(1-\frac{1}{x^{2}}\right) \ln (1+x)\right)\right]
$$

where $x=c / c^{*}$.

The excess of Rayleigh ratio $\Delta R(0)$ is given by:

$$
\frac{\Delta R(0)}{k^{\prime} c} \propto S(0) \bar{L}
$$

For micellar systems the average length $\bar{L}$ varyes with surfactant concentration according to 


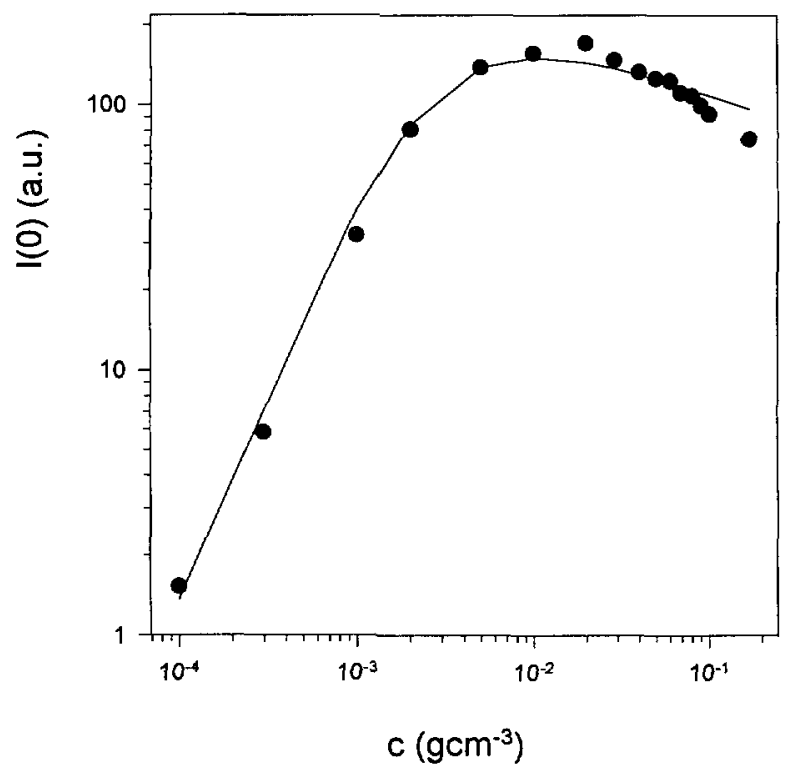

Fig. 15. - Effect of surfactant concentration on the scattered intensity extrapoled at zero- $q,[\mathrm{KBr}]=$ $0.5 \mathrm{M}, T=33.2^{\circ} \mathrm{C}$. The curve represents the best fit to the data of equation (27).

$\bar{L} \sim c^{1 / 2}$. The curve reported in Figure 15 is the best fit to the data of equations (26) and (27), where the two adjustable parameters are $c^{*}$ and the constant in front of the right side of equation (27). The best fit is obtained with $c^{*}=3.4 \times 10^{-3} \mathrm{gcm}^{-3}$. It can be seen that $c^{*}$ is smaller by a factor of $\sim 3$ than the concentration at which the scattered intensity is maximum, and of the same order as that corresponding to the maximum of the correlation lengths (cf. Fig. 8). The same approach has been used previously to analyse the light-scattering data in polymer-like reverse micelles by Schurtenberger and Cavaco [59]. In the fit of Figure 15, the effect of size polydispersity of micelles has not been taken into account. This would slightly modify the crossover concentration $c^{*}$ and also the shape of the curve in the dilute regime. Also the $c^{1 / 2}$ micellar length dependence was derived by using a mean field model. A scaling approach for semi-dilute solutions predicts a $c^{06}$ variation of $\bar{L}$ [30].

The effect of the temperature on the scattering properties in the semi-dilute range is also in agreement with the theoretical predictions made for semi-dilute solutions of classical polymers in good solvent. In the latter case $\xi$ can be written as $[38,47,56]$ :

$$
\xi=v^{-1 / 4} c^{-3 / 4} a^{-1 / 2}
$$

where $v$ is the excluded volume parameter and a the length of the statistical unit, that is the natural persistence length in the case of micellar systems. Here we have taken the Flory value $3 / 5$ for the exponent $\nu$ giving the size $R$ of one coil in a good solvent as a function of the polymerization index $N$. The only effect of $T$ on $\xi$ must be through the excluded volume parameter and/or the persistence length $\xi_{\mathrm{p}}$ of the micelle. The first effect leads indeed to a decrease of $\xi$ but it must be very weak since it is generally assumed that the good solvent is athermal with respect to the macromolecule conformation. However in micellar systems, the excluded volume parameter should be taken rather as a phenomenological parameter that takes into account a possible effect of ionic atmosphere. As for the effect of the persistence 
length it is also weak and acts in opposite direction since this parameter is given by [10]:

$$
\xi_{\mathrm{p}} \sim \frac{K_{\mathrm{e}}}{T}
$$

where $K_{\mathrm{e}}$ is an elastic constant.

Therefore only the increase of the excluded volume induced by an increase of temperature can explain the observed very weak decrease of the correlation length $\xi_{\mathrm{H}}$.

Finally, considering the effect of salt content, illustrated in Figure 11, the observed behaviour, that is, a decrease of both the hydrodynamic correlation length and the zero- $q$ scattered intensity upon increasing salt concentration is rather unexpected. According to equation (28), a decrease of the correlation length or equivalently an increase of the osmotic pressure can be produced by an increase of either the excluded volume parameter $\mathrm{v}$ or of the persistence length $\xi_{\mathrm{p}}$. It is rather unlikely that the salt could induce such effects as generally the addition of salt leads to a phase separation that is the signature of attractive interactions and therefore of a negative excluded volume parameter. Another interpretation is based on the possible formation of branched micelles at high salt concentrations suggested first by Porte et al. [60]. Such an effect has been suggested to interpret the results of rheological experiments $[24,25,34,35]$. We shall discuss this point in the next paragraph. The enhancement of the osmotic pressure by the polymer branching has been predicted theoretically [61] and found experimentally [12] in solutions of classical polymers. In this case, however, the comparison concerned a system of monodisperse linear polymers and a broad distribution of randomly branched polymers. The increase of the osmotic pressure for branched systems was due to the presence of small aggregates that were able to penetrate the big ones. Here we are dealing with a system that transforms upon addition of salt from a solution of polydisperse linear micelles to a solution of polydisperse branched micelles, the total micellar length being kept constant. The effect of such a transformation on the osmotic pressure is not trivial. In any case the experimental results of Figures 11 indicate without ambiguity that, if there is branching, it increases the osmotic pressure. One must also note that the systems at high salt contents exhibit a larger concentration dependence of the zero- $q$ scattered intensity (cf. Fig. 9).

5.2. Viscoelasticity. - The main conclusion to be drawn from the analysis of the slow mode is that the corresponding characteristic time is close to the terminal time of the stress relaxation, in good agreement with the theoretical predictions. Another interesting confrontation to the theory can be made by examing the behaviour of the relative amplitude $A_{\text {rep }}$ of the slow mode. According to equations (13) and (14) this amplitude depends only on the ratio $M_{\mathrm{g}} / K$ with a prefactor that is larger for $q R_{\mathrm{G}}>1$ than for $q R_{\mathrm{G}}<1$. In our experiments we observe a slight decrease of the amplitude of the slow mode upon increasing $q$. However, this effect is not very significant as the measured amplitude can take into account part of the Rouse contribution (that can vary with $q$ ) in addition to the reptational contribution. In this respect, we can remark that for worm-like micelles the terminal time of the stress relaxation and the Rouse time are closer than in classical polymers because of the breaking process that speeds up the relaxation.

Furthermore, if we assume that both moduli exhibit the same scaling as a function of surfactant concentration, we expect the relative amplitude at a given scattering angle be independent of $c$. This is what is observed within the experimental accuracy for the data in Table I, relative to systems with $0.5 \mathrm{M} \mathrm{KBr}$ and $T=33.2^{\circ} \mathrm{C}$.

The behaviour of $A_{\text {rep }}$ with the salt content is more surprising. One observes (Tab. I) a slight increase of $A_{\text {rep }}$ with [KBr] that suggests that the quality of the diluent becomes poorer. 
Table I. - Variation of the amplitude of the slow mode as a function of surfactant concentration, $\mathrm{KBr}$ concentration and scattering angle.

\begin{tabular}{|l|l|l|l||}
\hline $\mathrm{c}\left(\mathrm{g} / \mathrm{cm}^{3}\right)$ & {$[\mathrm{KBr}](\mathrm{M})$} & $\theta$ & $A_{,}(\%)$ \\
\hline 0.06 & 0.5 & 130 & 14.7 \\
0.07 & & & 14.5 \\
0.08 & & & 14.6 \\
0.09 & & & 14.8 \\
0.10 & & & 14.9 \\
0.12 & & & 15.6 \\
0.15 & & 130 & 15.6 \\
\hline 0.10 & 0.5 & & 14.9 \\
& 1 & & 174 \\
& 1.5 & & 170 \\
& 2 & & 18.7 \\
& 2.5 & 60 & 18.0 \\
\hline 0.10 & 0.5 & 90 & 17.2 \\
& & 130 & 16.2 \\
& & & 14.9 \\
\hline
\end{tabular}

This is coherent with the presence of attractive forces leading to the intermicellar branching but does not explain the behaviour of the osmotic pressure.

The variations of the relaxation time associated with the slow mode as a function of surfactant concentration, temperature and salt concentration are in agreement with the rheological results.

Finally we come back to the observation mentioned in the paragraph "Data Analysis" that in most cases we find an intermediate mode in the Contin procedure. According to the theory such a mode is predicted and is associated to the Rouse contribution. Indeed the characteristic time obtained experimentally, as in the exemple of Figure 7 is compatible with the Rouse process. Also, the small amplitude is in agreement with the prediction of equation (13). However, the experimental accuracy does not allow us to conclude unambiguously on that point.

\section{Conclusion}

The results presented in this paper show that the analogy between solutions of giant micelles and of polymer molecules stands not only for the static properties but also for the dynamical ones. In fact, the theoretical models describing the coupling of concentration fluctuations to viscoelasticity are better verified in micellar systems than in real polymer solutions. The behaviour of the slow relaxation process observed in DLS experiments as a function of temperature, salt and surfactant concentration parallels unambiguously that of the stress relaxation. 
In this respect, the light scattering technique appears to be very powerful to analyse the viscoelastic behaviour of micellar systems mainly when the relaxation time is too short to be measured easily by conventional rheological experiments. New experiments concerning this point will be presented in a forthcoming paper.

\section{Acknowledgments}

The authors thank F. Kern for his contribution to the rheological experiments.

\section{References}

[1] Cates M.E. and Candau S.J., J. Phys. Condens Matter 2 (1990) 6869, and references therein.

[2] Rehage H. and Hoffmann H., Mol. Phys. 74 (1991) 933, and references therein.

[3] Porte G., Appell J. and Poggi Y., J. Phys. Chem. 84 (1980) 3105.

[4] Candau S.J., Hirsch E. and Zana R., J. Phys. France 45 (1984) 1263.

[5] Candau S.J., Hirsch E. and Zana R., Collond Interface Scr. 105 (1985) 521.

[6] Candau S.J., Hirsch E. and Zana R., J. Physics of Complex and Supermolecular Fluids, S. Safran and N.A. Clark Eds. (Wiley, New York, 1987) 569.

[7] Appell J. and Porte G., Europhys. Lett. 12 (1990) 185.

[8] Jindal V., Kalus J., Pilsi H., Hoffmann H. and Lindner P., J. Phys. Chem. 94 (1990) 3129, and references therein.

[9] Vinson P. and Talmon Y., J. Collozd Interface Scr. 133 (1989) 288.

[10] Safran S., Turkevitch L. and Pincus P., J. Phys. Lett. 42 (1984) 1135.

[11] Hoffmann H., Platz G., Rehage H. and Schorr W., Adv. Collond Interface Scr. 17 (1982) 275.

[12] Lobl M., Thurn H. and Hoffmann H., Ber. Bunsen-Ges. Phys. Chem. 88 (1984) 1102.

[13] Hoffmann H., Löbl H., Rehage H. and Wünderlich I., Tenside Deterg. 22 (1985) 290.

[14] Thurn H., Löbl H. and Hoffmann H., J. Phys. Chem 89 (1985) 517.

[15] Shikata T., Hirata H. and Kotaka T., Langmuır 3 (1987) 1081; 4 (1988) 354; 5 (1989) 398.

[16] Shikata T., Hirata H., Takatori E. and Osaki K., J. Non-Newtonıan Flurd. Mech. 28 (1988) 171.

[17] Imae T., Abe A. and Ikeda S., J. Phys.Chem. 92 (1988) 1548.

[18] Rehage H. and Hoffmann H., J. Phys. Chem. 92 (1988) 4712.

[19] Candau S.J., Hirsch E., Zana R. and Adam M , J. Collovd Interface Sci. 122 (1988) 430.

[20] Candau S.J., Hirsch E., Zana E. and Delsanti M., Langmurr 5 (1989) 1525.

[21] Messager R., Ott A., Chatenay D., Urbach W. and Langevin D., Phys. Rev. Lett. 60 (1988) 1410.

[22] Kern F., Zana R. and Candau S.J., Langmurr 7 (1991) 1344.

[23] Kern F., Lemarechal P., Candau S.J. and Cates M.E., Langmuir 8 (1992) 437.

[24] Khatory A., Lequeux F., Kern F. and Candau S.J , Langmuir 9 (1993) 1456.

[25] Candau S.J., Khatory A., Lequeux F. and Kern F., J. Phys.IV France 3 (1993) 197.

[26] Kern F., Lequeux F , Zana R. and Candau S.J., Langmurr 10 (1994) 1714.

[27] Israelachvili J.N., Mitchell D.J. and Ninham B.W., J. Chem. Soc., Faraday Trans. II 72 (1976) 1525.

[28] Blankschtein D., Thurston G. and Benedeck G., J. Chem Phys. 85 (1986) 7268, and references therein.

[29] Appell J., Porte G. and Poggi Y., J. Collozd Interface Scı. 87 (1982) 492.

[30] Cates M.E., Macromolec. 20 (1987) 2289. 
[31] Cates M.E., Europhys Lett. 4 (1987) 497.

[32] Cates M.E., J. Phys. France 49 (1988) 1583.

[33] Granek R. and Cates M.E., J. Chem. Phys. 96 (1992) 4758.

[34] Appell J., Porte G., Kathory A., Kern F, and Candau S.J., J. Phys. II France 2 (1992) 1045.

[35] Khatory A., Kern F., Lequeux F., Appell J., Porte G., Morie N., Ott A. and Urbach W., Langmurr 9 (1993) 933.

[36] Lequeux F., Europhys. Lett. 8 (1992) 675.

[37] Semenov A.N., Physica A 166 (1990) 263.

[38] See for instance: Joanny J.F. and Candau S.J., in Comprehensive Polymer Sciences, Vol. 2, C. Booth and C. Price Eds. (Pergamon Press Oxford, 1989).

[39] Adam M. and Delsanti M., Macromol. 18 (1985) 1760.

[40] Brochard F. and De Gennes P.G., Macromol. 10 (1977) 1157.

[41] Brown W., Johansson K. and Almgren M., J. Phys. Chem., 93 (1989) 5888.

[42] Turner M. and Cates M.E., Langmuir 7 (1991) 1590.

[43] De Gennes P.G., J. Chem. Phys. 55 (1971) 572.

[44] Doi M. and Edwards S.F., J. Chem Soc. Faraday Trans II 74 (1978) 1789; 1802; 1818.

[45] Doi M. and Edwards S.F., The Theory of Polymer Dynamics (Clarendon Press, Oxford, 1986).

[46] De Gennes P.G., Macromol. 9 (1976) 587.

[47] De Gennes P.G., Scaling Concepts in Polymer Physics (Cornell University Press, Ithaca, 1979).

[48] De Gennes P.G., J. Chem. Phys. 72 (1980) 4756.

[49] Pincus P., J. Chem. Phys. 75 (1981) 1996.

[50] Binder K., J. Chem Phys. 79 (1983) 6327.

[51] Wang C.H., Macromol 25 (1992) 1524.

[52] Doi M. and Onuki A., J. Phys. II France 2 (1992) 1631.

[53] Zana R., Benraou M. and Rueff P., Langmurr 7 (1991) 1071.

[54] Koppel D.E., J. Chem. Phys. 57 (1972) 4814.

[55] Provencher S.W., Makromol Chem. 82 (1985) 632.

[56] De Cloızeaux J. and Jannink G., Les polymères en solution, leur modélisation et leur structure (Les Editions de Physique, Les Ulis, France, 1987).

[57] Faetibold E. and Waton G., langmurr in Press.

[58] Ohta T. and Oono Y., Phys. Lett. 89A (1982) 460.

[59] Schurtenberger P. and Cavaco C., J. Phys. II France 3 (1993) 1279.

[60] Porte G., Gomati R., El Haitamy O., Appell J. and Marignan J., J. Phys. Chem. 90 (1986) 5746.

[61] Daoud M. and Leibler L., Macromol 21 (1988) 1497.

[62] Munch J.P., Delsanti M. and Durand D., Europhys. Lett. 18 (1992) 577. 